\title{
Preliminary Study of Ultrasonic Structural Quality Control of Swiss-Type Cheese
}

\author{
J. J. Eskelinen, ${ }^{\star 1}$ A. P. Alavuotunki,† E. Hæggström, ${ }^{*}$ and T. Alatossava \\ *Department of Physical Sciences, and \\ †Department of Food Technology, University of Helsinki, FIN-00014, Helsinki, Finland
}

\begin{abstract}
There is demand for a new nondestructive cheesestructure analysis method for Swiss-type cheese. Such a method would provide the cheese-making industry the means to enhance process control and quality assurance. This paper presents a feasibility study on ultrasonic monitoring of the structural quality of Swiss cheese by using a single-transducer 2-MHz longitudinal mode pulse-echo setup. A volumetric ultrasonic image of a cheese sample featuring gas holes (cheese-eyes) and defects (cracks) in the scan area is presented. The image is compared with an optical reference image constructed from dissection images of the same sample. The results show that the ultrasonic method is capable of monitoring the gas-solid structure of the cheese during the ripening process. Moreover, the method can be used to detect and to characterize cheese-eyes and cracks in ripened cheese. Industrial application demands were taken into account when conducting the measurements.
\end{abstract}

Key words: ultrasound, Swiss cheese, quality control, cheese structure

\section{INTRODUCTION}

In the Finnish Emmental (Swiss-type) cheese manufacturing industry, the process and quality control (QC) techniques still rely on old sensory and destructive sampling techniques. Such techniques waste resources and material and suffer from limited capacity and sensitivity. These drawbacks motivate the need to develop new nondestructive evaluation (NDE) methods for cheese QC.

In Swiss cheese the crack formation occurs in the late ripening process (Walstra, 1991) and causes economic losses because the cheeses cannot be sold as top grade. A basic knowledge of the Swiss cheese manufacturing

Received February 10, 2007

Accepted May 15, 2007.

${ }^{1}$ Corresponding author: joona.eskelinen@helsinki.fi process and the most common defect types allows identifying needs and demands for a nondestructive QC method. The Emmental-type cheese ripening process is presented in Figure 1. Cheese eye formation is initiated during the preripening stage. The propionic acid fermentation and eye formation take place in the warm room and are followed by storage ripening phase when the propionic acid fermentation is ceased. The fermentation level is traditionally determined by monitoring the increase in cheese block height and by analyzing a cheese sample bored from the $80-\mathrm{kg}$ cheese block. The sample analysis can include evaluation of size and distribution of eyes as well as determination of the proteolysis level. The invasively tested cheese samples must be further processed to avoid microbial contamination. Replacing the old methods with an NDE testing system, therefore, has the following effects: 1) Cumbersome manual drilling is no longer needed. Work is eased and savings in time, resource and storage usage accrue; 2) Product quality is more homogeneous due to more extensive sampling, which can cover the whole production batch; 3) Process control is eased. If deviations are detected, fast online measurements provide short response time to make process modifications.

Currently, defective cheeses are separated as late as in the cutting and packing line and are redirected to the processed cheese or grated cheese production. The late QC wastes storage ripening capacity and decreases packing line capacity. Online QC would alleviate these drawbacks and allow grated and processed cheese production to be based solely on low-quality cheeses, in contrast to preallocating whole (possibly prime grade) batches.

\section{Potential Online Monitoring Points}

There are several points in the Swiss-type cheese production chain where NDE inspection can generate added value (see Figure $1 \mathrm{~A}-\mathrm{C}$ ). At the end of the propionic acid fermentation (A), the NDE method could provide information about the size, density, and distribution of cheese-eyes as well as the physical properties of the cheese matrix. This information can be used to 


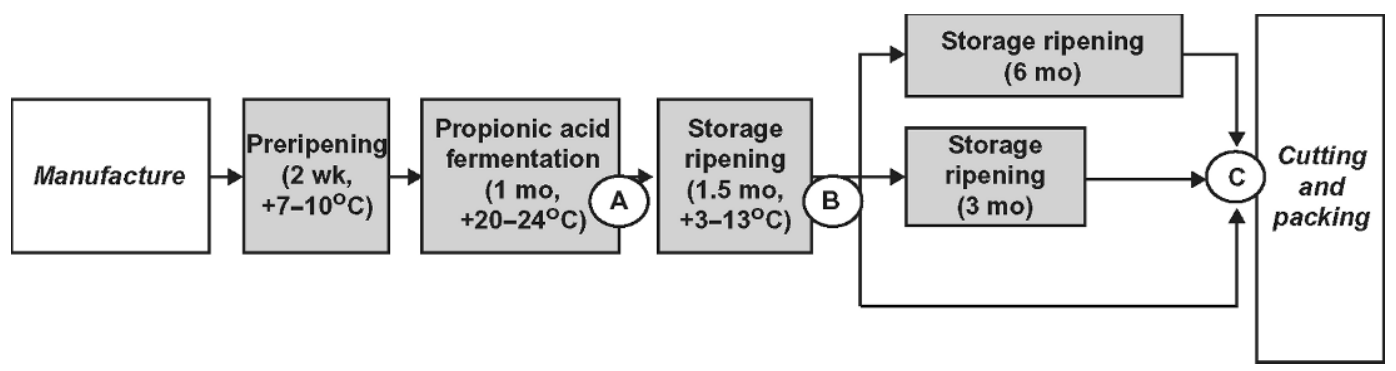

Figure 1. Emmental-type cheese ripening process (Kosikowski and Mistry, 1997; Fröhlich-Wyder and Bachmann, 2004) with typical ripening times applied in Finland. Panels A-C depict potential process phases for nondestructive monitoring..

determine the correct moment to stop the propionic acid fermentation. In case of defects or insufficient eye formation, process control measures can be executed. After the 1.5-mo storage ripening (B) period, the cheese susceptibility to cracking defects could be determined by evaluating the elastic properties of cheese and the presence of microcracks. Only prime grade cheese would be directed to further ripening and the cheeses susceptible to cracking would be cut, packed, and sold. The last monitoring point could be prior to cutting and packing $(\mathrm{C})$. Production volumes could be enlarged by cutting out small defect volumes at the packing line.

\section{Review of Potential NDE Techniques}

Efforts to develop NDE techniques for cheese QC have been reported. Infrared spectroscopy technologies can monitor free amino acids (Skeie et al., 2006), fat, moisture, and protein content of cheese (Adamopoulos et al., 2001), but infrared technologies are unable to monitor the inner structure due to their low $(\sim 1 \mathrm{~mm})$ penetration depth. Microwaves have been suggested for online monitoring of cheese moisture level (Horsfield et al., 1996). However, as imaging modality, microwaves tend to provide low resolution due to long wavelength (>1 cm). Currently, terahertz (T-ray) imaging is illsuited for large cheese-block imaging due to high water molecule absorption (Siegel, 2004). Moreover, T-ray imaging demands complex hardware. X-ray computer tomography can be used to detect foreign bodies in food products (Ogawa, et al., 1998), and x-ray techniques have been incorporated in food process automation $\left(\mathrm{X}^{4}\right.$, Loma-Cintex, Farnborough, UK). Magnetic resonance imaging (MRI) has been used to evaluate eye formation and structural quality (Rosenberg et al., 1992; Duce et al., 1995) of Swiss-type cheese. However, x-ray and MRI-based installations are expensive, costly to operate, and require sophisticated maintenance, making the cost per individual QC scan high. Moreover, they do not readily allow single surface scanning.
Conventional sensory tapping or acoustical (Benedito et al., 2006a) techniques cannot provide precise information about individual structural elements. Rheological characterization of cheese has also been conducted (Tovar et al., 2004), but rheological measurements are unsuitable for structural imaging applications. The fraction of cheese-eyes has been monitored with image analysis from optical dissection images (Caccamo et al., 2004). This method, like all optical methods, probes solely the surface and can thus be used merely during cutting and packaging on cut cheese blocks.

Ultrasonic methods hold a well-established position in nondestructive structural analysis. Ultrasonic imaging methods, common in medical and NDE applications, are normally based on pulse-echo measurement of sound reflection or scattering. The ultrasonic technique possesses characteristics that are advantageous compared with the aforementioned techniques. It is especially sensitive to detecting cavities (cheese eyes) and fissures, similar to acoustic contrast agents (Cosgrove, 2006). Compared with x-ray or MRI methods, ultrasonic devices are simple, fast, cheap, and small-making the cost per structural image low.

\section{Ultrasonic Cheese Characterization}

Previous work on ultrasonic cheese characterization mainly relies on through-transmission measurements of the longitudinal mode sound propagation velocity in bulk cheese. The properties of different cheese types have been studied ultrasonically in hard and semihard cheeses, such as Cheddar, Mahon, Manchego, and Parmiggiano-Reggiano.

Ultrasonic methods have been used to study milk renneting and coagulation (Taifi et al., 2006; Wang et al., 2007) and to monitor cheese maturation state and mechanical properties, such as deformability modulus, akin to Young's modulus (Benedito et al., 2000, 2006b). Pulse-echo single-surface cheese probing has been exploited in attempts to detect cracks in solid semihard 
and hard cheese (Orlandini and Annibaldi, 1983; Albrecht et al., 1999; Benedito et al., 2001) and the presence of foreign bodies in cheese (Haeggstrom and Luukkala, 2001). An air-coupled, noncontact through-transmission method has been used to determine sound propagation velocity, slice thickness (Saggin and Coupland, 2001), mechanical properties (Cho and Irudayaraj, 2003a), and foreign objects in Cheddar cheese (Cho and Irudayaraj, 2003b). However, using ultrasound to monitor the structural elements of eye-forming cheeses (such as the Swiss and Maasdammer type) has not been reported.

In this paper, we examine the potential of ultrasonic method to provide information about structural elements, such as eyes and cracks. We compare an ultrasonic image of the cheese interior to an image constructed from dissection photographs of the same cheese sample. In this visual evaluation, the type and the location of the structural formations are compared between the images. This study is a preliminary step to determine the feasibility of an ultrasound based QC method for Swiss cheese online monitoring.

\section{MATERIALS AND METHODS}

\section{Design Considerations}

Several conditions were imposed to simulate an industrial environment. Of the standard ultrasonic techniques for cheese QC; that is, pulse-echo and throughtransmission, the pulse-echo technique was investigated because online application restrictions favor single-surface cheese probing. Moreover, this method should provide better spatial resolution, especially in axial direction, than the through-transmission technique. The measurements were conducted through a commonly used maturation wrapper using only water coupling to meet the online situation restrictions.

\section{Measurement System}

The measurement set-up (see Figure 2) consisted of a single transducer $(2 \mathrm{MHz}, \mathrm{S} 12 \mathrm{~W} 2$, Karl Deutsch Prüf- und Messgerätebau GmbH, Wuppertal, Germany), pulser/receiver (Panametrics 5072PR, Panametrics Inc., Waltham, MA), oscilloscope (LeCroy 9310A, LeCroy Corporation, Chestnut Ridge, NY) and a computer with RS-232 and GPIB-interfaces. The transducer was moved with a computer-controlled translation stage (iselautomation $\mathrm{GmbH}$, Eichenzell, Germany) across the top surface of the stationary cheese. Constant loading and orthogonal insonication were ensured by attaching the transducer rigidly to a linear stage and by applying weights ( $40 \mathrm{kPa}$ induced pressure on cheese surface). The sample temperature was con-

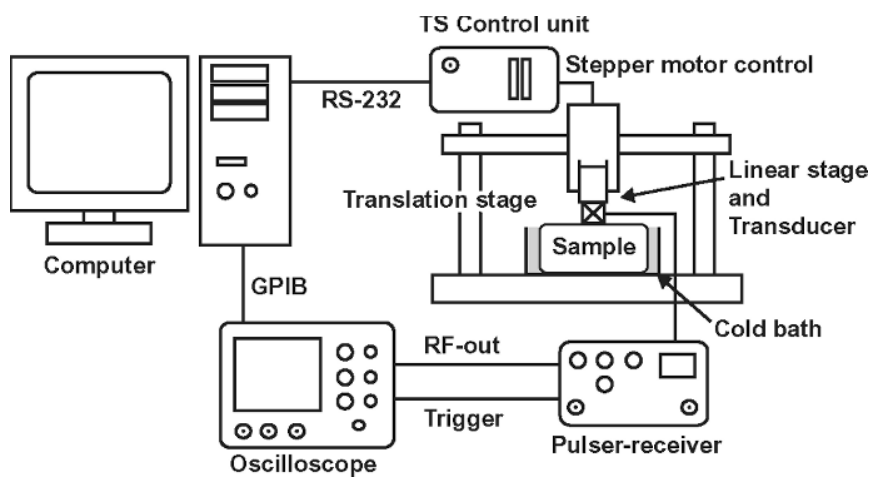

Figure 2. Schematics of the measurement set-up.

trolled by an ice-water mixture cold-bath, and tap water was constantly applied manually to the cheese surface to ensure coupling.

The $5072 \mathrm{PR}$ pulser was used in pulse-echo mode (energy 4, damping 5, HPF $1 \mathrm{MHz}$, and LPF $10 \mathrm{MHz}$ ) with a moderate $37-\mathrm{dB}$ amplification, to reduce ringing time present with extensive amplification. From each scan step, 6 radio frequency (RF) traces (A-lines) were read to the computer from the oscilloscope (across the GPIB-bus) and averaged. The time per scan step was $3 \mathrm{~s}$. Each averaged trace was recorded on the computer and off-line signal processing, and image rendering was conducted with Matlab (The MathWorks Inc., Natick, MA). In the defect signal analysis, a 1-MHz transducer (A303S, Panametrics Inc.) was used, and received signals were 100 -times averaged without high-pass filtering. Otherwise, the set-up was identical to the raster scan set-up.

\section{Signal Processing and Image Formation}

To construct an image from the raw RF traces, 5 signal processing steps were carried out. The raw signal of each RF trace was time-gated. Each trace was then enveloped with the Hilbert transform (Gammell, 1981) to allow plotting an intensity chart. A 100 -point moving average smoothed the data and suppressed noise. Then a prerecorded background was subtracted from each trace to reduce ringing effects and to extract unwanted features that appeared in every trace. Finally, timegain compensation (an exponential growing amplification as a function of depth) compensated for sound attenuation. After these steps the data were rendered into a 3D image. No interpolation between the voxels was used.

\section{Optical Image Formation from Photographed Cheese Slices}

To evaluate the performance of the proposed ultrasound method, an optical reference image was created. 
The ultrasonically characterized cheese sample was sliced into $3.3 \pm 0.4 \mathrm{~mm}$ (thick) vertical slices with a cheese-cutting knife. The slices were photographed using a basic flatbed scanner (300 dpi, Epson Perfection 1640SU, Epson America Inc., Long Beach, CA). The obtained images were treated in Adobe Photoshop (Adobe Systems Inc., San Jose, CA). The images were converted to gray-scale (0-256), the contrast and brightness were adjusted to emphasize the desired structure elements, the resolution was lowered to 20 $\mathrm{dpi}$, and the images were cropped (to obtain same image volume as in the ultrasonic image). The resulting bitmaps were then rendered into a 3D image with Matlab.

\section{Cheese Samples}

The experiments were conducted with samples of Emmental (Swiss-type) cheese. The samples, provided by Valio Ltd., were standard 6-kg blocks cut from matured 40 and $80 \mathrm{~kg}$ production blocks at the cutting line. The samples were ripened for 2 wk at 7 to $10^{\circ} \mathrm{C}$, for $4 \mathrm{wk}$ at 20 to $24^{\circ} \mathrm{C}$, and for 2 to $12 \mathrm{mo}$ in storage conditions, 4 to $7^{\circ} \mathrm{C}$.

Sample preparation included cutting the cheese blocks into smaller samples $\left(\sim 20 \times 10 \times 10 \mathrm{~cm}^{3}\right)$ with a cheese knife, and vacuum packing (5 to $10 \mathrm{mbar}$, Multivac C100, Multivac Sepp Haggenmüller GmbH\& Co, Wolfertschwenden, Germany) them with common plastic maturation wrapper (110 $\mu \mathrm{m}$ tubular bag EMVAC-DAE 5/110 F9, Sudpack Verpackungen GmbH, Ochsenhausen, Germany). The storage temperature for the cheese blocks and the samples was 4 to $5^{\circ} \mathrm{C}$.

\section{Ultrasonic Raster Scan}

A single transducer raster scan was conducted on a 15-mo matured Emmental cheese sample to obtain a $3 \mathrm{D}$ image of the sample interior and to evaluate the imaging system performance. The scan area was chosen because it was suspected to contain crack and eye structures. The area was limited to $6 \mathrm{~cm} \times 4 \mathrm{~cm}$, and the scan mesh size was $0.75 \pm 0.004 \mathrm{~mm} \times 0.75 \pm 0.004$ $\mathrm{mm}$. The probing depth was windowed from 2.5 to 7 $\mathrm{cm}$, and the data were processed as described in the methods section. The scan depth was windowed due to signal-to-noise restrictions (no good reflections were detected deeper from the sample). The measurement was conducted at $8 \pm 2^{\circ} \mathrm{C}$.

\section{RESULTS AND DISCUSSION}

\section{Ultrasonic and Optical Images of Emmental Cheese Sample}

Constructed ultrasonic (raster scan) and optical images of the 15-mo ripened Emmental sample are pre-

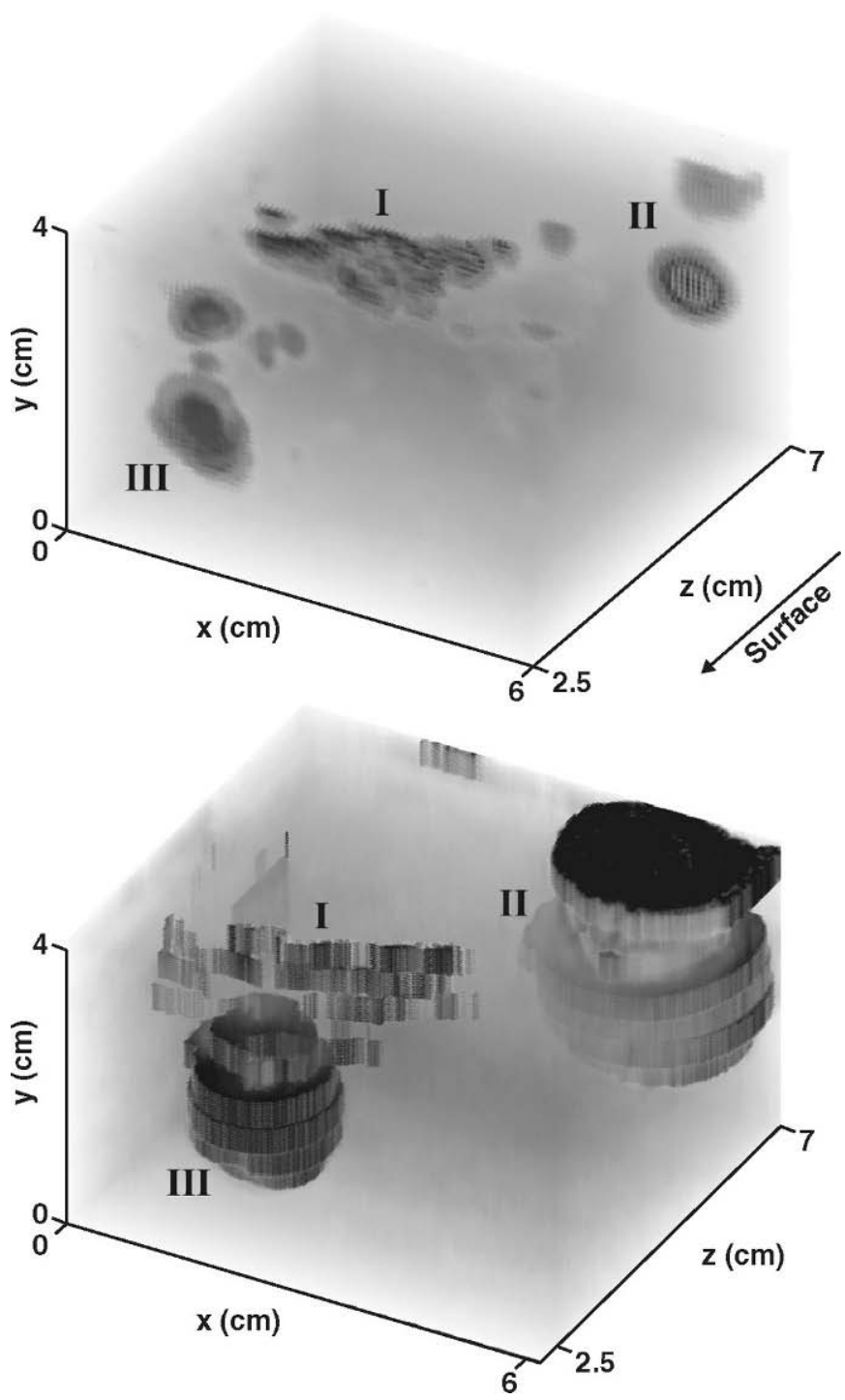

Figure 3. Two different images on the same cheese sample interior obtained with the ultrasonic method (top) and reconstructed from the photographed slices (bottom). Corresponding structural components (I: crack, II: two cheese-eyes, III: one cheese-eye) are indicated in both figures.

sented in Figure 3. Figure 4 illustrates 2 sample slices used to construct the $3 \mathrm{D}$ optical image. The ultrasonic image featured 3 cheese-eyes (diameter $\varnothing=1$ to $2 \mathrm{~cm}$ ) visible at depths of 3 to $7 \mathrm{~cm}$ and one crack visible at $4 \mathrm{~cm}$ depth. Moreover, there was a smaller crack region visible at $3 \mathrm{~cm}$ depth. In signal transmission tests it was possible to obtain a reflection from the opposite cheese surface at $20 \mathrm{~cm}$ distance. Furthermore, $\varnothing=1 \mathrm{~cm}$ cheese-eyes generated a detectable reflection at depth of $10 \mathrm{~cm}$. 


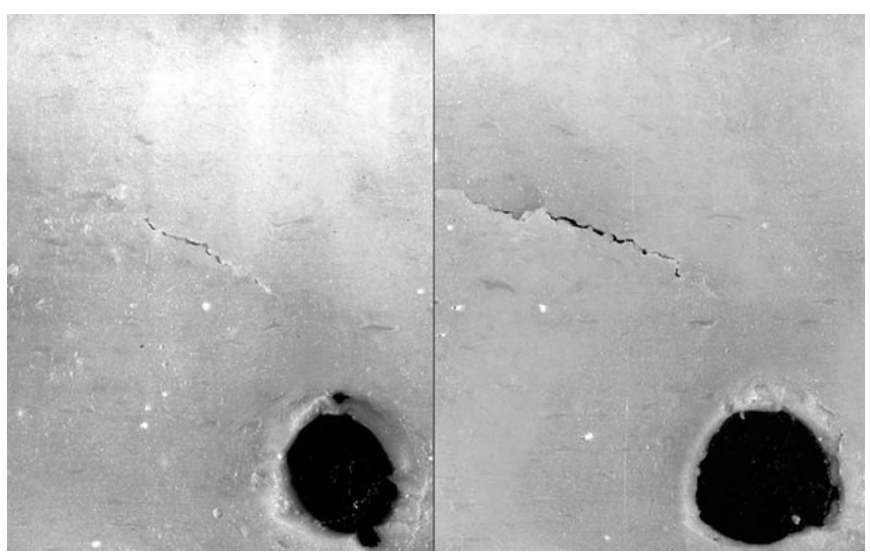

Figure 4. Two slices of the Emmental cheese sample used in ultrasonic and optical image studies.

\section{Evaluation of Correspondence of Ultrasonic and Dissection Images}

The ultrasonic 3D image featured similar types of structural formations as seen in the optical reference image (Figure 3) with corresponding locations. Two different cavity types could be distinguished from the bulk cheese. The eye cavities appear in the ultrasonic image as round planes and the crack formation as a ragged plane running almost parallel to the surface. The cracks could be detected and differentiated from the eye cavities due to their irregular and distinctive shape. Moreover, it could be seen that bulk cheese regions were relatively clear in the ultrasonic image. The resolution of the imaging system is a function of the probing depth, scan mesh size, transducer focus, and aperture, as well as the capability of signal processing algorithms. No experimental evaluation of the imaging resolution or method sensitivity was conducted during this phase of the project. However, the resolution of the obtained ultrasonic images allowed recognizing basic structural elements like eyes and cracks as well as differentiating between them. The results indicated that this technique provides potential to achieve adequate imaging resolution to allow structural QC of Swiss-type cheese online.

The optical reference method suffers from some weaknesses that should be considered when comparing the 2 images. First, the y-axis resolution of the image was poor due to scarce slicing $(>3 \mathrm{~mm})$. Second, the shear forces induced by the cutting blade might have promoted crack formation by extending the cracks, which makes them appear bigger in the optical image than they actually were. Third, the flat bed scanning will not reproduce the smallest heterogeneities in the cheese matrix. For example, tiny cracks would not have produced enough optical contrast to appear in the scanned dissection pictures. This could explain why the smaller reflection areas visible in the ultrasonic image were not visible in the optical 3D image. The 3D ultrasonic image was obtained at a slightly different scan angle than the cutting angle in the optical image. This altered the relative position of the structural elements in the $3 \mathrm{D}$ pictures.

\section{Defect Detection}

To achieve good defect detection, correct classification of the structural elements must be established. Image analysis can be used to analyze the structures. For instance, shape recognition algorithms can be exploited to differentiate between cracks and round cheese eyes in the ultrasonic images. Moreover, structure size can be evaluated from the image. Image analysis, combined with pattern analysis of RF signal waveforms, provides enhanced defect monitoring.

Examples of 1-MHz RF-waveforms together with the photographs of the corresponding samples are presented in Figure 5. These waveforms demonstrate that the reflection from a cheese eye was almost ideal, whereas the crack-reflection was distorted due to interference between diffuse reflections. Normally formed cheese eyes feature a glossy (acoustically flat) surface in contrast to rough crack surfaces. Whey nests feature numerous small cavities $(\varnothing \leq 3 \mathrm{~mm})$, which act as individual scatterers (the sound wavelength, $\lambda$, in cheese at $1 \mathrm{MHz}$ is $\lambda \approx 1.7 \mathrm{~mm}$ ). This results in a highly distorted reflection from several multiple scattered signals. These differences can be exploited for structure classification and defect detection. Current computing power allows online RF signal analysis. For instance, nonlinear spectral analysis could allow detecting small splits and cracks (Ohara et al., 2007).

\section{Benefits and Drawbacks}

Due to the high reflection coefficient from solid-gas interfaces, only the uppermost cavity surface on the probing axis can be seen with multi-transducer pulseecho probing. However, this shadowing effect could be reduced by using a multitransducer trapezoid delay line or a phased array technique, which allows beam focusing and steering as well as faster scanning.

Only part of the spherical cheese-eyes are perpendicular to the transducer probing axis, meaning that only a portion of the reflected intensity is detected. This complicates eye-detection and must be accounted for when estimating eye size. Moreover, ultrasonic attenuation in Emmental cheese is substantial (up to $5 \mathrm{~dB} /$ $\mathrm{cm}$ at $2 \mathrm{MHz}$ ), which lowers penetration depth and reduces signal-to-noise ratio. Problems rising from attenuation effects need to be overcome to achieve fast 


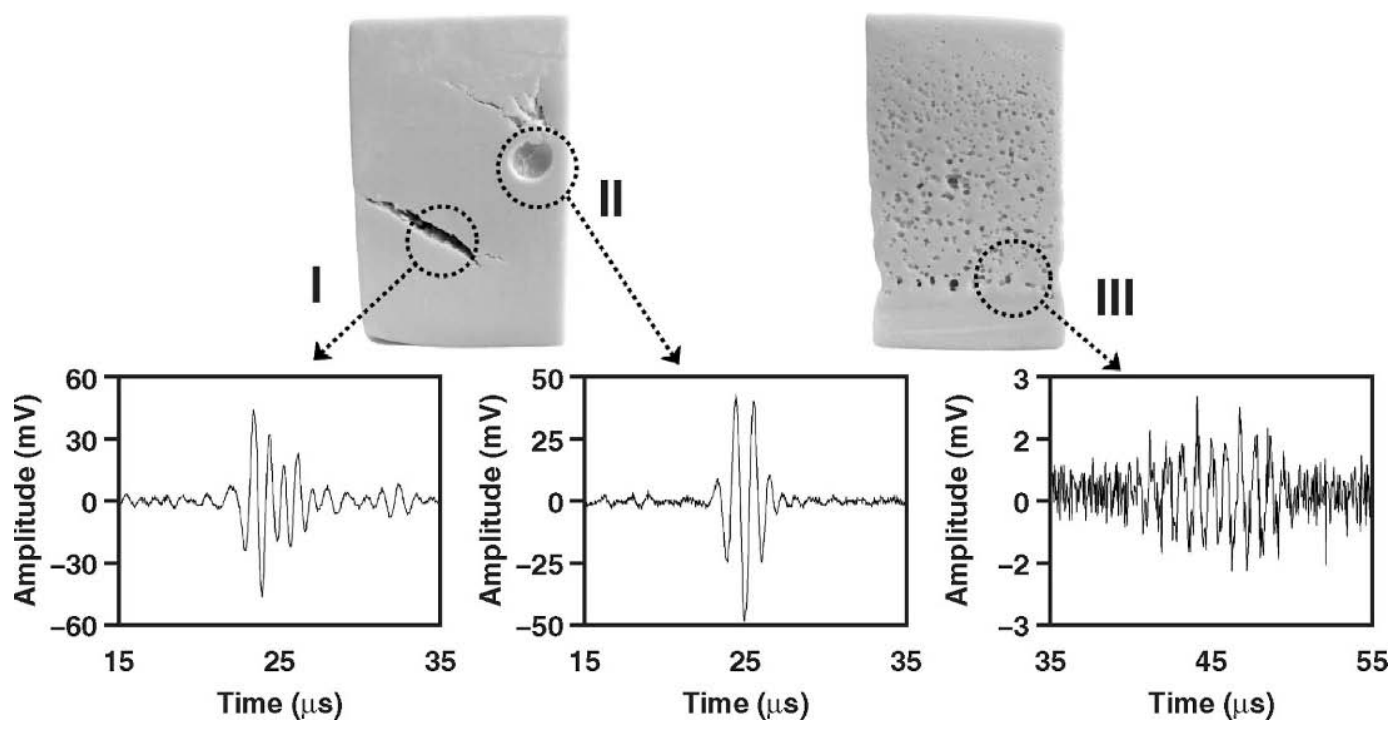

Figure 5. Ultrasonic reflections $(1 \mathrm{MHz}, 100$ times averaged) from different structural elements found in 2 Swiss cheese samples (20 $\mathrm{cm} \times 10 \mathrm{~cm} \times 10 \mathrm{~cm}$ ). Dotted circles and arrows indicate the measured structural elements and their corresponding radio-frequency signals. Characteristic signals from a crack (I) and an eye (II) in a 12-mo-old sample (left). Whey nest structure (III) in a 4-mo-old sample (right).

scanning of the entire cheese volume. However, cheese shows similar soft-structure behavior as tissue, indicating that medical ultrasonic imaging modalities can be exploited in cheese structure characterization.

Preliminary sound velocity measurements indicated that the ultrasound propagation velocity increases as a function of ripening time. This could be exploited for maturation state estimation. Moreover, ultrasonic methods are versatile-they can monitor cheese maturation state, presence of foreign bodies, as well as structural defects. All these tasks could be performed by a single inexpensive device.

\section{CONCLUSIONS}

The aim of this study was to evaluate the feasibility of the proposed ultrasonic method to provide relevant information for quality and process control as a part of Swiss cheese manufacture. This feasibility was demonstrated by comparing a 3D ultrasound image and a reference 3D image created from the same cheese sample. Eyes, cracks, and bulk cheese matrix could be differentiated in the ultrasound images. The structural elements in the ultrasonic image showed spatial conformity with the image constructed from the sliced cheese sample. Commercially relevant structural elements were identifiable at depths that should allow singlesurface probing of entire cheese blocks.

These results indicate that the ultrasonic method can be used to aid in Swiss-type cheese process and quality control. The main foreseen applications are structural
QC (defect detection) and eye formation monitoring, in which the endpoint for the propionic acid fermentation could be determined. The method also provides potential for ripening stage monitoring and for foreign body detection. The defect detection sensitivity for QC purposes can be further improved by applying a combination of various methods (e.g., signal pattern analysis and image analysis).

However, the verification of the online capability of the proposed ultrasonic method still demands more work and experiments. For online application, the most critical factors are scan speed vs. scan volume (penetration depth), method sensitivity, image resolution, and the impact of eye shadowing.

\section{ACKNOWLEDGMENTS}

We acknowledge development manager Tapani Kivelä and vice president of product development Matti Harju at Valio Ltd. and Sakari Juurus from the Foundation of Finnish Cheese Producers for their valuable comments that guided our efforts throughout the project. The Foundation of Finnish Cheese Producers and the Finnish Emmental cheese producers Valio Ltd. and JK Juustokaira Ltd. provided grants to support this study.

\section{REFERENCES}

Adamopoulos, K. G., A. M. Goula, and H. J. Petropakis. 2001. Quality control during processing of feta cheese: NIR application. J. Food Compos. Anal. 14:431-440.

Albrecht, B., H. R. Ulmann, and J. O. Bosset. 1999. Ultraschallechografie, nachweis von rissen in käselaiben-Ultrasound 
echography: Detection of cracks in cheeses. DMZ-Lebensmittelindustrie und Milchwirtschaft 120:268-272.

Benedito, J., J. Carcel, G. Clemente, and A. Mulet. 2000. Cheese maturity assessment using ultrasonics. J. Dairy Sci. 83:248-254.

Benedito, J., J. Carcel, M. Gisbert, and A. Mulet. 2001. Quality control of cheese maturation and defects using ultrasonics. J. Food Sci. 66:100-104.

Benedito, J., T. Conde, G. Clemente, and A. Mulet. 2006a. Use of the acoustic impulse-response technique for the nondestructive assessment of Manchego cheese texture. J. Dairy Sci. 89:44904502.

Benedito, J., S. Simal, G. Clemente, and A. Mulet. 2006b. Manchego cheese texture evaluation by ultrasonics and surface probes. Int. Dairy J. 16:431-438.

Caccamo, M., C. Melilli, D. M. Barbano, G. Portelli, G. Marino, and G. Licitra. 2004. Measurement of gas holes and mechanical openness in cheese by image analysis. J. Dairy Sci. 87:739-748.

Cho, B. K., and J. M. K. Irudayaraj. 2003a. A noncontact ultrasound approach for mechanical property determination of cheeses. J. Food Sci. 68:2243-2247.

Cho, B. K., and J. M. K. Irudayaraj. 2003b. Foreign object and internal disorder detection in food materials using noncontact ultrasound imaging. J. Food Sci. 68:967-974.

Cosgrove, D. 2006. Ultrasound contrast agents: An overview. Eur. J. Radiol. 60:324-330.

Duce, S. L., M. H. G. Amin, M. A. Horsfield, M. Tyszka, and L. D. Hall. 1995. Nuclear magnetic resonance imaging of dairy products in two and three dimensions. Int. Dairy J. 5:311-319.

Fröhlich-Wyder, M. T., and H. P. Bachmann. 2004. Cheeses with propionic acid fermentation. Pages 141-156 in Cheese: Chemistry, Physics and Microbiology-Vol. 2: Major cheese groups. P. F. Fox, P. L. H. McSweeney, M. T. Cogan, and T. P. Guinee. 3rd ed. Elsevier, London, UK.

Gammell, P. M. 1981. Improved ultrasonic detection using the analytic signal magnitude. Ultrasonics 19:73-76.

Haeggstrom, E., and M. Luukkala. 2001. Ultrasound detection and identification of foreign bodies in food products. Food Contr. 12:37-45.

Horsfield, B., J. A. R. Ball, J. R. Holdem, R. B. Keam, W. S. Holmes, and A. Green. 1996. On-line moisture measurement during cheese production. http://www.is.irl.cri.nz/pubdoc/1996/RMIT96.pdf Accessed June 30, 2007.

Kosikowski, F. V., and V. V. Mistry. 1997. Cheese and fermented milk foods. Procedures and analysis. Vol. II. 3rd ed. F. V. Kosikowski, Westport, CT.

Ogawa, Y., K. Morita, S. Tanaka, M. Setoguchi, and C. N. Thai. 1998. Application of X-ray CT for detection of physical foreign materials in foods. Trans. ASAE 41:157-162.

Ohara, Y., T. Mihara, R. Sasaki, T. Ogata, S. Yamamoto, Y. Kishimoto, and K. Yamanaka. 2007. Imaging of closed cracks using nonlinear response of elastic waves at subharmonic frequency. Appl. Phys. Lett. 90:011902. doi:10.1063/1.2426891

Orlandini, I., and S. Annibaldi. 1983. Investigation about new techniques for the evaluation of Parmigiano-Reggiano cheese structure: Ultrasounds and X rays. Sci. Tecn. Latt. Cas. 34:20-30.

Rosenberg, M., M. McCarthy, and R. Kauten. 1992. Evaluation of eye formation and structural quality of Swiss-type cheese by magnetic resonance imaging. J. Dairy Sci. 75:2083-2091.

Saggin, R., and J. N. Coupland. 2001. Non-contact ultrasonic measurements in food materials. Food Res. Int. 34:865-870.

Siegel, P. H. 2004. Terahertz technology in biology and medicine. Microwave theory and techniques. IEEE Trans. 52:2438-2447.

Skeie, S., G. Feten, T. Almøy, H. Østlie, and T. Isaksson. 2006. The use of near infrared spectroscopy to predict selected free amino acids during cheese ripening. Int. Dairy J. 16:236-242.

Taifi, N., F. Bakkali, B. Faiz, A. Moudden, G. Maze, and D. Décultot. 2006. Characterization of the syneresis and the firmness of the milk gel using an ultrasonic technique. Meas. Sci. Technol. 17:281-287.

Tovar, C. A., I. Franco, E. Carballo, M. J. Camara, J. Carballo, and C. A. Cerdeirina. 2004. Influence of the ripening time on the viscoelastic behaviour of Tetilla cheese. Food Sci. Technol. Int. 10:305-313.

Walstra, P. 1991. Rheological foundation of eye and slit formation. Bull. IDF 268:65-66.

Wang, Q., S. Bulca, and U. Kulozik. 2007. A comparison of lowintensity ultrasound and oscillating rheology to assess the renneting properties of casein solutions after UHT heat pre-treatment. Int. Dairy J. 17:50-58. 